\title{
Hybrid techniques to improve face recognition based on features extraction methods and Haar discrete wavelet transformation
}

\author{
Israa AbdulAmeer AbdulJabbar Zainab Ali Yakoob \\ University of Technology, Department of Computer Science, Iraq, \\ Baghdad
}

Recived : $25 \backslash 1 \backslash 2018$

Revised : 41212018

Accepted : $7 \backslash 2 \backslash 2018$

Available online : $20 / 2 / 2018$

DOI: 10.29304/jqcm.2018.10.2.372

\begin{abstract}
This paper uses hybrid techniques to improve the rate of recognition for a face from identified data set of faces. These techniques are summarized by applying firstly the Haar discrete wavelet transformation method in order to enhance and compress the images of the data set and store the results for each process in a separate data set. Secondly, applying a hybrid method from two popular face recognition methods called Principal Component Analysis and Singular Value Decomposition for extracting feature from the images. This work applied by using a dataset contains 400 images for 40 different persons called Olivetti Research Laboratory (ORL). In calculating the distances between image vectors, Manhattan measurement is used and its show a very good results in recognition rate. From this work, it can be concluded that recognition rate increments with the decrementing in the number of dataset images and increasing the threshold value. The expended time in execution decreases in a very obvious way when using the compressed dataset rather than the enhanced dataset which its images has four times the size of the images in the compressed dataset.
\end{abstract}

Keywords: Face recognition, Principal Component Analysis (PCA), Singular Value Decomposition (SVD),Haar Discrete Wavelet Transform (HDWT) and Manhattan distance.. 


\section{Introduction}

The aging of information grows up so quickly with enormous amount of data and the needs to transform all paper works to electronic work has led to the development of communication networks together with the spread of internet around the whole world rising the need to perform more security ways to prevent the mischievous using them and protect the user information [1]. Scientists are thinking of using human individual biological features which cannot be denied, lost or stolen; so they are use extracting features from images methods and classification techniques to present new methods [2].

From these needs, the face recognition techniques which are a subfield from pattern recognition were presented and preferred than other biometric because it is the only type which allows one to many identification like the case of catching terrorist in a crowd place such as terminals of airport or train [3]. The main tasks of face recognition are identification and verification [4]. The essential methods of face recognition are holistic, feature-based and hybrid between the first two methods and each of them has a specific function [5][6].

Wavelet is used in various fields such as engineering, sounds study, computer vision, picturing, face recognition, processing of images and etc. Wavelet accuracy depends on the shift and scale of signals and it characteristic has been developed to deal with high-frequency and lowfrequency of signals to provide the coefficients of wavelet [7]. Also, it is used in images enhancement in order to smooth data and bring higher resolution component to be analyzed or can be used in case of compression. [8].

Face recognition has been developed in the last era and many methods were presented and hybrid with each other or with other techniques such as discrete wavelet transforms to gain best recognition ratio with high performance and less execution time.

The main drawback in the previews works are did not mention some of basic operations of the main used techniques in detail like how to compute the eigenvectors in principal component analysis method or how to choose the threshold value. The goal of this paper is to improve the rate of recognition using various methods of recognition and de-noise the data set images and compress them in other data set.
Section 2 will show a literature survey for many researchers; section 3 display hybrid techniques to improve face recognition in detail, section 4 presents the results and discussion and section 5 provides the conclusion.

\section{Literature Review}

In 2013, Sodhi 1 and Madan published a paper in image recognition using Principal Component Analysis (PCA) based on various techniques like Discrete Wavelet Transform (DWT) to extract image feature by analyzing image to multi-level of scaling and used the approximation sub-band level which has the low frequency features (LL) with various distance classifier. Level 1, 2 and 3 used to get recognition ratio equal to 77,79 and 78 respectively using Euclidean distance and got $73 \%$ at all the levels using city-block distance. This paper did not mention detail about the threshold values, number of training images used or time expending in execution [9].

In 2014, Esraa and Et. al. applied HDW to denoise the images using Olivetti Research Laboratory (ORL) dataset and enhance them using "Laplacian of Gaussian filter" in order to improve recognition rate. In this paper the time execution and threshold value did not mentioned [10].

In 2016, Thamizharasi with his partner Jayasudha were written a research to improve face recognition using two procedures to provide integration between them to solve the lighting pre-processing problem. The used dataset in this paper was ORL which firstly enhanced by applying 2D discrete wavelet on them. In the second stage applied a method named Contrast Limited Adaptive Histogram Equalization after that they got the ratio of recognition using Gabor wavelet and they found the best output of these methods appears with images have problem in lighting. They gained 98.50 recognition rate using ORL dataset. Unfortunately the used number of eigenvectors, the threshold value and the expended time did not mentioned in this paper [11].

In 2017, Abdullah, Jeaid and R. Hussein used SVD to get the feature form faces and artificial neural network to recognize these faces into their models. The researchers also applied discrete wavelet on the dataset to compress the image face and reduce the time expended in execution, their work produce recognition rate equal to $95 \%$ but there is no mention to the time or the threshold value [12]. 
Israa .A/Zainab .A

\section{Hybrid techniques to improve face recognition}

\subsection{Haar Discrete Wavelet Transform}

It is one of discrete family wavelet transforms methods used in feature extraction and works at frequency domain [13]. It has effective tool to maintain the energy of the image and great tool to understand the meaning of discrete wavelet. It is simple because it use addition and subtraction operations rather than multiplication and can be completed in a short time. Its robust shows in edge extraction, image coding, binary logic design and compression. Size of vector should be even number raise to power of two [14].

Process of Haar depends on split vector with $\mathrm{N}$ length into two parts. The first N/2 row takes pair of numbers at a time and computes the average addition for them and putting it in the first half output vector as in equation 1 .

$\mathrm{a}_{\mathrm{n}}=\frac{\mathrm{f}_{2 \mathrm{n}-1}+\mathrm{f}_{2 \mathrm{n}}}{\sqrt{2}} \quad, n=1,2 \ldots N / 2$

The second N/2 rows take pair of numbers at a time and calculate the average difference for them and putting it into the second half output vector with a factor for weight equal to $\sqrt{2}$ as in equation 2 .

$d_{n}=\frac{\mathrm{f}_{2 \mathrm{n}-1}-\mathrm{f}_{2 \mathrm{n}}}{\sqrt{2}}, \quad n=1,2 \ldots N / 2$

Haar filter is used to get the average from original image named low pass filter $h=$ $\left(h_{0}, h_{1}\right)=\left(\frac{\sqrt{2}}{2}, \frac{\sqrt{2}}{2}\right)$ which is used for the first half of rows. The filter is chosen according to ability of reproduce the same two numbers that are reversed each other. Notice that the summation of these two numbers of filter is equal to $\sqrt{2}$.

For the second half of rows uses high pass filter which is $g=\left(g_{0}, g_{1}\right)=\left(-\frac{\sqrt{2}}{2}, \frac{\sqrt{2}}{2}\right)$ where return values near to zero if the numbers are almost the same otherwise the filter returned best weight from these two numbers. Two levels of decomposition with traditional wavelet transform where $\mathrm{L}$ means Low pass and $\mathrm{H}$ means High pass filter as show in figure 1 [15].

\begin{tabular}{|l|l|l|}
\hline LL1 & LH1 & \multirow{2}{*}{ LH } \\
\cline { 1 - 2 } HL1 & HH1 & \\
\cline { 1 - 1 } HL & \multirow{2}{*}{ HH } \\
\hline
\end{tabular}

Fig.(1): Two level decomposition with traditional wavelet transform [16]

In order to apply Haar Discrete Wavelet Transform (HDWT) on images with two dimensions, it should convert the image into $2 \mathrm{D}$ array with size $\mathrm{N} * \mathrm{M}$ where $\mathrm{N}$ is rows number and $\mathrm{M}$ is columns number. The process is done by multiplying columns matrix by the original image matrix then the output is multiplied by the transformed rows matrix as defined in equation 3 [17]:

$Y=W_{M} A W_{N}^{T}$

Four quarters are resulted in form of image constructed from high and low pass filters of Haar where the upper left corner holds the average image which contains the most important information about the image, while the upper right corner contains horizontal detail of image. The lower left corner detect vertical detail and lower right corner detects diagonal details [17]. The output matrix is shown as a follows:

$$
\begin{aligned}
Y=W A W^{T}= & {\left[\begin{array}{l}
H \\
G
\end{array}\right] A\left[\begin{array}{l}
H \\
G
\end{array}\right]^{T}=\left[\begin{array}{c}
H A \\
G A
\end{array}\right]\left[\begin{array}{c}
H^{T} \\
G^{T}
\end{array}\right] } \\
& =\left[\begin{array}{ll}
H A H^{T} & H A G^{T} \\
G A H^{T} & G A G^{T}
\end{array}\right]
\end{aligned}
$$

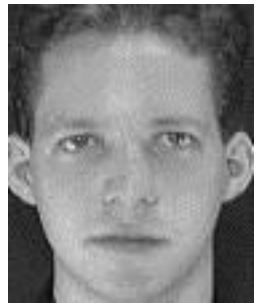

(a)

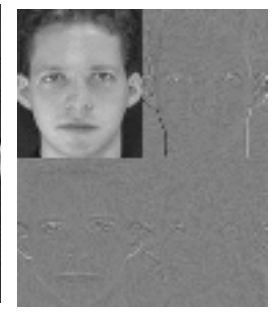

(b)

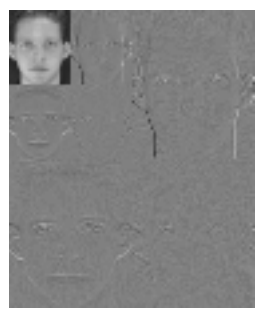

(c)
Fig. (2): Haar wavelet process 
In figure 2 shown (a) is the original image and (b) is the transformed level 1 Haar image and (c) is level 2 Haar image.

The blurred upper corner portion from the level one (L1) as shown in figure 2.b where the transformed image has the most intensities of the original image which are shown in figure 2.a so, repeating HWT process can be continued on this portion only and the output from the second transforming process can be replaced into the upper left portion from level one (L1) image as shown in figure 2.C. Many levels can be obtained from repeating HWT until the desired output gained.The inverse HWT inverted the previous process by applying onto the blurred portion until getting the original image [18]. Figures 3 and 4 show two dimensions of discrete Haar/inverse wavelet transform process.

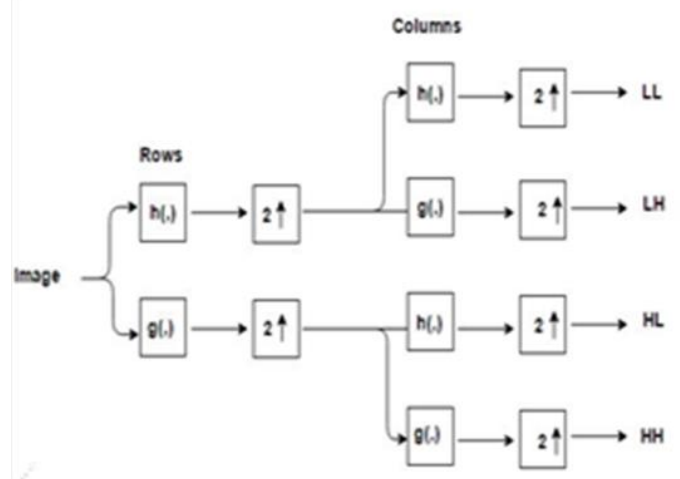

Fig.(3): 2D discrete wavelet transform[19]

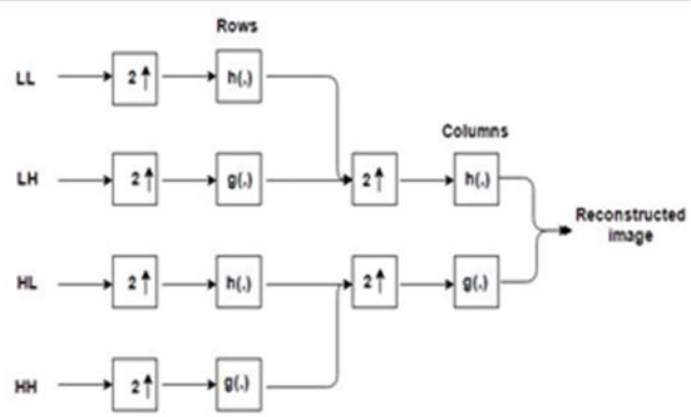

\subsection{Principal Component Analysis (PCA):}

PCA is a powerful statistical approach used in recognizes the faces, coding and compression. It is classified as holistic method approach. PCA very useful with images have different poses but it is considered as time consuming approach [20]. The basic advantage of PCA is dimensionality reduction which removes the redundant data by computing variance between the data and getting the covariance matrix which it gives knowing about how much these data are correlated. The mathematical operation used in PCA is dot product [11].

PCA approach applied by the following steps [9][10][21][22]:

- Read dataset and convert images

$$
\begin{aligned}
& \text { to } \left.\overrightarrow{a_{1}}, \overrightarrow{a_{2}}, . . \overrightarrow{a_{M}}\right)
\end{aligned}
$$

- Calculate the mean face $(\bar{A})=$ $\frac{1}{M-1} \sum_{i=0}^{M-1} A_{i}$

- Subtracted mean face from image vector $\phi=A-\bar{A}$

- Calculate covariance matrix $\mathrm{C}=$ $\frac{1}{M-1} \sum_{i=0}^{M-1} A^{T} A$

- Calculate eigenvalue and eigenvectors from $\mathrm{C}$ and sort eigenvectors according to its eigenvalues descending. $\operatorname{eig}(\mathrm{C})=\mathrm{V} \alpha$

- Calculate eigenfaces (U) by project (V) from small space to large space $U=A V$

Size of $U$ matrix equals to $\left(x^{*} y, K\right)$, where $\mathrm{K}$ is a value of used eigenvectors [23].

Fig.(4): Iinverse HaarDiscrete Wavelet[19] 
The first eigenvalues $(\alpha)$ related to its eigenvectors (V) have the high energy merged from the corresponding eigenvectors in small eigenspace so select eigenvectors done by pick the highest eigenvalues to gain eigenfaces in large space.

- Calculate eigenvectors weights

$\Omega$ for the training dataset $\Omega_{(K, K)}=U^{T} \phi$

- Calculate the distance between vectors $£_{K}=\left\|\Omega_{i}-\Omega_{K}\right\|$

Manhattan distance was used in this paper which gets absolute difference between weights. Where $\mathrm{k}=$ higher values of eigenvalues

$$
\sum_{i=0}^{k}\left|x_{i-} y_{i}\right|
$$$$
\text { Manhattan Distance }(x, y)=
$$

- Calculate threshold which acts as the maximum distance between all the vectors.

Threshold=t* $\max \left\|\Omega_{i}-\Omega_{K}\right\|$

Next steps processed on loaded test images:

- Load image and convert it into vector $\left[X_{p}\right]$.

- Calculate mean test image $\bar{X}=\sum_{i=0}^{P-1} x_{i}$

- $\quad$ Subtract $X$ from mean test image $\phi_{p}=X_{p}-\bar{X}_{p}$

- Calculate weight image in each eigenvectores $\Omega_{K}=U^{T} \phi$

- Calculate minimum distance from weight testing image to weights of training images $£$ $=\min \left\|\Omega-\Omega_{K}\right\| \quad$,Where $\Omega$ is test image weight and $\Omega_{k}$ the weight of training images.

- Output test image is known face or unknown.

\subsection{Singular Value Decomposition (SVD)}

In statistic PCA and in order to minimize data space of two dimensions from large space to small space, Singular Value Decomposition (SVD) has an active part in this process by project the data image from large space to small space. SVD processed on 2D images matrix A to gain three matrices which are orthogonal on each other. Where $U_{(n, m)}$ matrix in a large space, $V_{(m, m)}$ is orthogonal matrix in a small space and $\Lambda_{(\mathrm{m}, \mathrm{m})}$ is diagonal matrix contain the singular values and the other matrix elements are zeros [20]. Calculating singular value decomposition for matrix $A_{(n, m)}$ where it must satisfy the conditions $\mathrm{n}=\mathrm{x} * \mathrm{y}$ and $n>m$ has the decomposing form:

\section{SVD Algorithm[21]}

Begin

Step1: Convert $\mathrm{N}$ training set into $\mathrm{A}$ matrix with size $\mathrm{M}=$ (rows * columns).

Step2: Calculate average face $\bar{A}$.

Step3: Subtract average face from matrixA.

Step4: Calculate the coordinate vector $\mathrm{Y}=\mathrm{A}^{\mathrm{T}} /(\mathrm{N}-1)$.

Step5: Calculate singular value for matrix $\mathrm{Y}, \operatorname{SVD}(\mathrm{Y})=\mathrm{U} \Lambda \mathrm{V}$.

Step6: Project the test image onto eigen faces to compute recognition rate.

End

\section{The Proposed Hybrid System}

In the proposed system, thw first stage is examining the dimensions of the image in the dataset which should be equal to a number 2 rise to power of $\mathrm{N}\left(2^{N} * 2^{N}\right)$ without any remainder because the equation in the transformation algorithm needs to be in an even number for rows and columns. 
Then load the dataset of ORL with 400 images and analyze each image into its essential colors which are red, green and blue and store them into three matrices. The elements of the matrix are defined as double to keep the correct value for the colors accurate without any distortion. The three matrices will be used and their values processed by various algorithms in all the upcoming stages separately and in the last stage, the three colors values will be combined to each other to represent the new processed pixel and display the processed test image recognized or not.

The second stage is applying Haar transformation algorithm of discrete wavelet and its inverse algorithm in order to make enhancement on the images and the output will be compressed and enhanced images. The Haar process depends on calculate the average and difference for each row and column. Haar transformed the images according to a specific chosen level of transformation to gain the output image as in figure 2 . The inverse Haar wavelet must applied with the same level of transformation for the input image in order to have the enhanced image. This process applied on all the images on the dataset and stores the result in a specific enhanced dataset. The loaded images have dimensions equal to $(92 * 112)$ so at level one of transformation, the output average image has the most amount of information from the image with a quarter size of the original image where the size of original image equals to $(92 * 112)$ while the compressed image has size $\left(46^{*} 56\right)$ as shown in figure 5 .
The compressed image will be stored in other compressed images dataset and will be used in recognition process which gaves a very good result in a half time expended in recognition the original image.
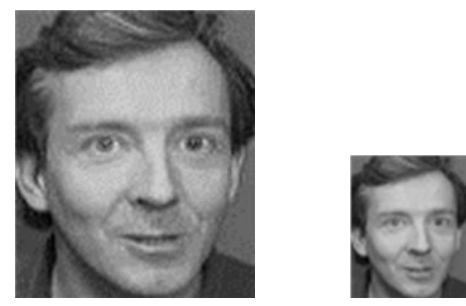

(a)Original image (b)Compressed image

Fig (5): Original image and compressed image

In the third stage a hybrid algorithm of Singular Value Decomposition (SVD) method and Principal Component Analysis (PCA) method was proposed to improve face image recognition as shown in figure 6 which represents the block diagram of the proposed work.

In PCA, dimensionality reduction for the covariance matrix provide execution in less time because it reduces the dimension in very active way.

$$
\begin{aligned}
& \mathrm{C}=\mathrm{A}^{\mathrm{T}} * \mathrm{~A} \\
& (400 * 400) \quad(400 * 10304)(10304 * 400)
\end{aligned}
$$

As a result from converting the images into $\mathrm{A}$ matrix of vectors, the dimension of A represent as height the size of each image which is 10204 and the width represent the number of images in the dataset. After reducing the covariance matrix with smaller size $(400 * 400)$ then it is easier to calculate the eigenvalue and eigenvectors for covariance matrix. Hybrid SVD with PCA allows getting the better features from the image vectors to enhance and raise the rate of recognition by has the benefit of PCA in dimensionality reduction and the speed of processing the covariance matrix in SVD in the small space because the (SVD) uses the coordinate matrix which has size $(400 * 10304)$ in order to have the eigenvalue and eigenvectors as follow: 


$$
\begin{aligned}
& \mathrm{Y}=\mathrm{A}^{\mathrm{T}} \quad /(\mathrm{N}-1) \\
& (400 * 10304) \quad(400 * 10304)
\end{aligned}
$$

This process slow because the vector has size 10304 and for its mathematical operation required to obtain the determinant and eigenvalues in the Covariance matrix $(C)$. SVD in execution treat as fast in processing so when it operate on a vector has size 400 rather than 10304 will process vectors in less time. Other distinction between the two methods is sorting the eigenvectors depending on its related eigenvalues and chooses best eigenvectors, while all the eigenvectors used in the SVD method without take into account the best eigenvector which takes a lot of time as well using this part of the way of PCA is the best. The other reason is with SVD when calculate the distance between each eigenvector will take a lot of time because it takes all the eigenvectors while in the PCA method calculate the distance only between the best eigenvectors.

In hybrid mode PCA SVD tried to get the best output with different recognition ratio by getting the highest eigenfaces for all 400 images in the ORL database recognizing the image of the input test image. The recognition rate depends on the number of faces are recognized from 400 and dividing the number gained over 400.In this paper all 400 images are tested on 400 images of training set until the hybrid mode algorithm is repeated 400 times to have the recognition rate, execution time was calculated and registered and notice that its be higher with few seconds when the eigenvector number and threshold value are rise but recognition rate was incremented.

\section{Hybrid (PCA SVD) Algorithm}

Input: $\mathrm{N}$ Training set enhanced images/compressed images by Haar discrete wavelet Output: Recognition face ratio Begin

Step 1: Load training images data set;

Step 2: Read $m=$ number of training image;

Step 3: Read $\mathrm{x}=$ =image_width,$\quad \mathrm{y}=$ image_height;

Step 4: Convert each training image to vector

Step 5:Normalize all images vector in matrix A.

Step 6: Calculate average face image $\phi=A-\bar{A}$.

Step 7: Calculate covariance $\mathrm{C}=\frac{1}{\mathrm{M}-1} \sum_{\mathrm{i}=0}^{\mathrm{M}-1} \mathrm{~A}^{\mathrm{T}} \mathrm{A}$.

Step 8: Calculate $S V D(C)=U \Lambda V$.

Step 9: Calculate weight for each eigen face $\Omega_{\mathrm{K}}=\mathrm{U}^{\mathrm{T}} \phi$.

Step 10: Calculate distance between eigen face vectors $£=\min \left\|\Omega-\Omega_{K}\right\|$.

Step 11: Load test image.

Step 12: Calculate sum $=\sum_{\mathrm{i}=0}^{\mathrm{M}-1} P_{i}$

Step 13: Calculate average test image vector pixels=each image pixel $\operatorname{sum} /(x * y)$

Step 14: Calculate the minimum distance.

Step 15: Recognize test image is face or not and calculate recognition rate.

End. 
Fig (6): The proposed work block diagram

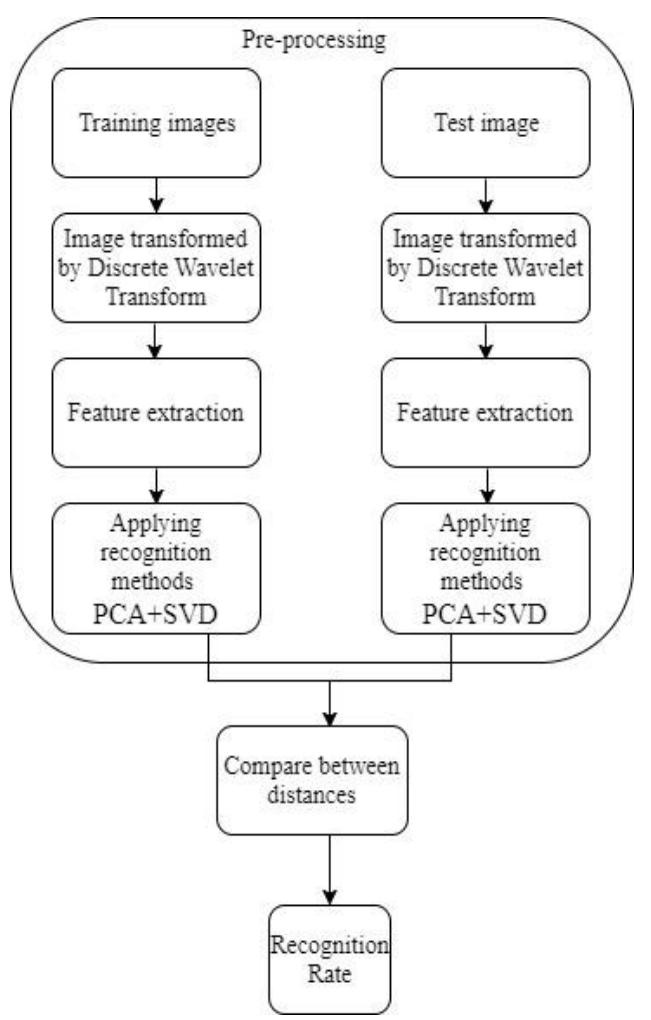

\section{Experimental Results and Discussion}

The proposed work of hybrid PCA and SVD methods applied using enhanced and compressed data set by Haar discrete wavelet. The proposed work experimental results registered using different cases in order to get best results. Various threshold values are used with various numbers of training set images and selecting distinct number of eigenface to get the best result with better performance evaluations.

The proposed system depends, in the first place, on the enhancement using wavelet transformation for all the images in the data set. The required time to enhance all the images in the database (for example the ORL database) was so limited, where it takes for executing Haar wavelet and its inverse in less of one minute. The resulted data set was stored inside the system folder and submitted to the user control. The enhanced data set only can be accessed by authorized user for using the system.

As mentioned before; the proposed system merges PCA and SVD methods. the results obtained by testing various threshold values, different number of training set and different number of eigenfaces in order to have the best results.

The number of images in the training set is a powerful tool in control the ratio of recognition where the rate decrement with increasing in the number of images while the rate increment with the decreasing of number of images. Choosing higher number of threshold value leads to rise the recognition rate as described in table (1).

Time parameter is a very remarkable factor in the recognition process, table (2) shows the recognition rate with the original size of image and the time consumed which it reduced in an obvious way when using the compressed dataset in the process of recognition as shown in table (3) and it can be noticed the difference in time between table (2) and table (3).

The hybrid of the PCA and SVD resulted with better recognition rate than using each method of them separately as shown in table (4).

Table (1): Recognition rate results using various number of training

\begin{tabular}{|l|l|l|l|}
\hline Distance & \multicolumn{2}{|c|}{ Trainin } \\
method & $\begin{array}{l}\text { Threshol } \\
\text { get set } \\
\text { number }\end{array}$ & $\begin{array}{l}\text { Recognitio } \\
\text { d }\end{array}$ & Rate \\
\hline Manhatta & 400 & 0.3 & 96.5 \\
\cline { 2 - 4 } n distance & 200 & 0.3 & 97.5 \\
\cline { 2 - 4 } & 400 & 0.5 & 99.75 \\
\cline { 2 - 4 } & 200 & 0.5 & 100 \\
\hline
\end{tabular}


Table (2): Recognition rate results using 400 ORL training images

\begin{tabular}{|l|l|l|l|l|}
\hline $\begin{array}{l}\text { Distance } \\
\text { method }\end{array}$ & Threshold & $\begin{array}{l}\text { K } \\
\text { number } \\
\text { of } \\
\text { eigenvect } \\
\text { ors }\end{array}$ & RR & $\begin{array}{l}\text { Execution } \\
\text { time } \\
\text { in seconds }\end{array}$ \\
\hline \multirow{5}{*}{$\begin{array}{l}\text { Manhattan } \\
\text { distance }\end{array}$} & $\mathbf{0 . 3}$ & 40 & $\mathbf{8 5 . 7 5}$ & $\mathbf{3 1 . 4 6 5 2 5}$ \\
\cline { 2 - 5 } & $\mathbf{0 . 5}$ & $\mathbf{4 0}$ & $\mathbf{9 7 . 2 5}$ & $\mathbf{2 2 . 2 4 1 0 8}$ \\
\cline { 2 - 5 } & $\mathbf{0 . 5}$ & $\mathbf{6 0}$ & $\mathbf{9 6 . 5}$ & $\mathbf{3 8 . 4 1 5 0 9}$ \\
\cline { 2 - 5 } & $\mathbf{0 . 7}$ & $\mathbf{1 0 0}$ & $\mathbf{9 9 . 7 5}$ & $\mathbf{3 7 . 3 1 5 2 6}$ \\
\cline { 2 - 5 } & $\mathbf{0 . 8}$ & $\mathbf{2 0 0}$ & $\mathbf{1 0 0}$ & $\mathbf{5 1 . 6 8 8 7 2}$ \\
\hline
\end{tabular}

Table (3): Recognition rate results using average face part only from Haar level 1

\begin{tabular}{|l|l|l|l|}
\hline $\begin{array}{l}\text { Distance } \\
\text { method }\end{array}$ & $\begin{array}{l}\text { Trainin } \\
\text { g Set }\end{array}$ & $\begin{array}{l}\text { Recogniti } \\
\text { on Rate }\end{array}$ & $\begin{array}{l}\text { Time } \\
\text { execution } \\
\text { (s) }\end{array}$ \\
\hline \multirow{2}{*}{$\begin{array}{l}\text { Manhatta } \\
\text { distance }\end{array}$} & 100 & 100 & 2.612205 \\
\cline { 2 - 4 } & 200 & 100 & 5.008010 \\
\cline { 2 - 4 } & 300 & 100 & 11.308830 \\
\cline { 2 - 4 } & 400 & 97.25 & 16.040918 \\
\hline
\end{tabular}

Table (4) Comparative between literature survey and proposed work

\begin{tabular}{|l|l|l|}
\hline Reference & Methods & RR \\
\hline$[9]$ & PCA+DWT & $\mathbf{7 3 \%}$ \\
\hline$[10]$ & PCA+HDWT & $\mathbf{9 7 . 1 4 \%}$ \\
\hline$[11]$ & $\begin{array}{l}\text { DWT+Gabor } \\
\text { Wavelet }\end{array}$ & $\mathbf{9 8 . 5 \%}$ \\
\hline$[12]$ & SVD+ANN+DWT & $\mathbf{9 5 \%}$ \\
\hline $\begin{array}{l}\text { Proposed } \\
\text { work }\end{array}$ & PCA+SVD+HDWT & $\mathbf{1 0 0 \%}$ \\
\hline
\end{tabular}

\section{Conclusions}

In this paper, the proposed system introduces a way to recognize an image contains a face as a known or unknown face in a specific data set which has many identified images enhanced or compressed by Haar wavelet transform where recognition process has been processed using a hybrid of two techniques called PCA and SVD. The combination of Haar wavelet transform with face recognition methods provides the advantage of the two techniques to make a very integrated system that can give best performance. Different threshold values were used in this work and concluded that increasing its value has helped in raising the accuracy rate and that is normal because threshold value represented proportion amount from the maximum distance between any two image vectors and when increasing this proportion and that means the possibility for test images to be in the image space was high. Reducing the number of training set images has led to increase the accuracy rate of recognition and it decreased the time of execution.

\section{References}

[1] Brindha T. and Josephine MS. ,(2015), "Facial Feature Recognition Using Biometrics", Proceedings of 27th IRF International Conference.

[2] Urvashi B. , Rohit S. ,(2014)" A Survey On Face Detection Methods And Feature Extraction Techniques Of Face Recognition", IJETTCS, Vol. 3, Issue 3. [3] Deepika G. and Anubhav K. S.,(2012)" Face Recognition", IOSR Journal of Engineering (IOSRJEN), Vol. 2, Issue 7, PP. 128-133.

[4] Sandeep Mishra, Anupam Dubey,(2015) "Face Recognition Approaches: A Survey", International Journal of Computing and Business Research (IJCBR),Vol. 6 Issue 1.

[5] Divyarajsinh N. Parmar and Brijesh B. Mehta,(2013), "Face Recognition Methods \& Applications", ,Int.J.Computer Technology \& Applications, Vol. 4 (1),PP.84-86.

[6] Ghanshyam Sahu, Asstt. Prof. Bhanupratap Singh, Asstt. Prof. Pradeep Tripathi,(2014), "A Comprehensive Review on Face Recognition Techniques", International Journal of Emerging Technology and Advanced Engineering, Vol. 4, Issue 6. 
[7] Valens C. ,"A Really Friendly Guide to Wavelets", tutorial, available at http://perso.wanadoo.fr/polyvalens Lclemens/wavelets/wavelets.html. [8] Barford, L.A.,. , Fazzio R. S. and David R. S. ,(1992), “An Introduction to Wavelets", Instruments and Photonics Laboratory, HPL-92-124.

[9] Kuldeep S. S. and Madan L. ,(2013), "Comparative Analysis of PCA-based Face Recognition System using different Distance Classifiers", International Journal of Application or Innovation in Engineering \& Management, Vol. 2, Issue 7.

[10] Isra'a Abdul-Ameer Abdul-Jabbar, Jieqing Tan, Zhengfeng Hou.,(2014), "Two Adaptive Image Pre-processing Chains for Face Recognition Rate Enhancement". International Journal of Multimedia and Ubiquitous Engineering,Vol. 9, No.3 ,pp.379-392.

[11] A. Thamizharasi and Jayasudha J. S.,(2016)," A Hybrid Method for Face Recognition using LLS CLAHE Method", International Journal of Computer Applications, Vol. 152 ,No.7.

[12] M N Abdullah,H A Jeiad and R A.Hus (2017), "Multibiometric Identification System based on SVD and Wavelet Decomposition", Engineering and Technology Journal ,Vol. 35, Part A, No. 1.

[13] Bedi S. S. and Khandelwal R.,(2013)," Comprehensive and Comparative Study of Image Fusion Techniques", International Journal of Soft Computing and Engineering, Vol.3, Issue 1.

[14] Anisha A. and Swati M. , (2014), ” Comparative Analysis Of Haar and Skin color Method For Face Detection", ICRAIE.
[15] Anuj B. and Rashid A. ,(2009)," Image Compression Using Modified Fast Haar Wavelet Transform", World Applied Sciences Journal, Vol. 7 No.5, PP. 647-653.

[16] Kyanda S. K. and Feng C. ,(2012),” Multiresolution Techniques and 2D Signal Application", IMACS, Vol I, march 14-16.

[17] Fliege N. J. ,(2004), "Multirate digital signal processing", Jogn Wiely and Sons,New York.

[18] Jaideva C. G.and Andrew K. C. ,(2010), " Fundamentals of Wavelets: Theory, Algorithms, and Applications, Second Edition", "filter bank and discrete wavelet transform" chapter 7 .

[19] Abdul-Jabbar I. A.,(2014), "face recognition and matching enhancement based on wavelet transform", PHD thesis, Hefei university of technology. [20] Aluko J. O. , Omidiora E. O. , Adetunji A. B. , Odeniyi O. A. , (2015),"Performance Evaluation of Selected Principal Component AnalysisBased Techniques For Face Image Recognition", International Journal Of Scientific \& Technology Research Vol. 4, Issue 01.

[21] Rashmi Ravat, Namrata Dhanda,(2015)," Performance Comparison of Face Recognition Algorithm Based on Accuracy Rate", International Journal of Advanced Research in Computer and Communication Engineering Vol. 4, Issue 5.

[22] Turk M. and Pentland A. ,(1991), "Eigenfaces for Recognition", Journal of Cognitive Neuroscience, vol. 3, no. 1. [23] Shang-Hung Lin, Ph.D.,(2000)," An Introduction to Face Recognition Technology", informing science special issue in multimedia informing technologies Vol. 3, No.1 


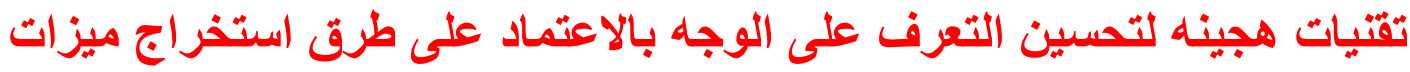

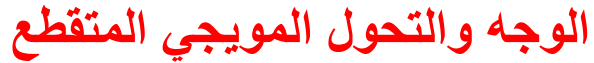

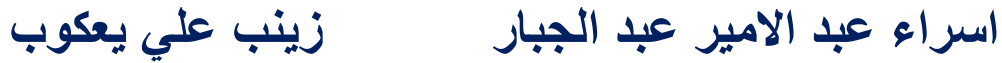 \\ الجامعة التكنولوجية ، قسم علوم الحاسوب الحبر ، العراق ، بغداد
}

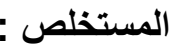

هذا البحث يقوم باستخدام تقنيات لتحسين تمبيز الوجوه من قاعدة بيانات معرفة مسبقا. هذه التقنيات تلخص او لا

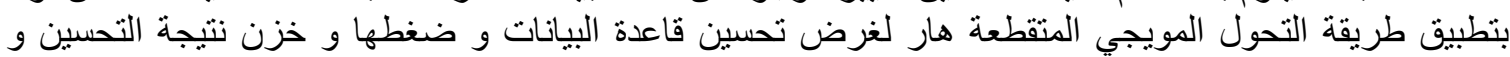

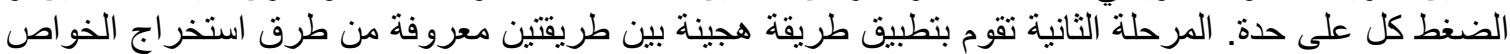

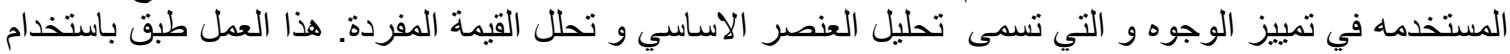

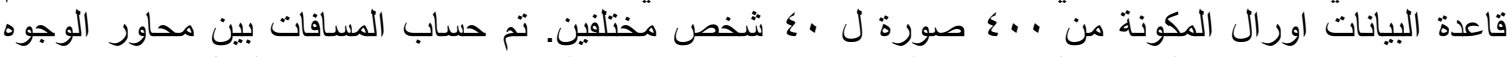

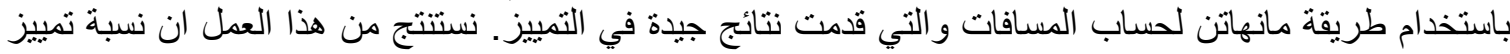

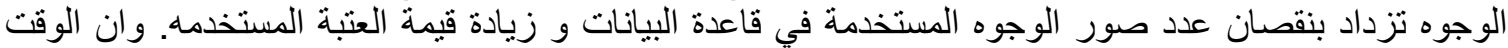

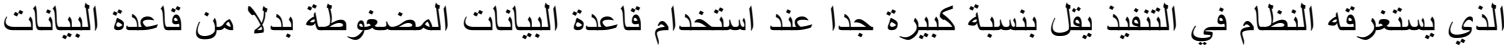
المحسنة و ذلك لان حجم الصور فيها يكون اكبر باربع مرات من حجم الصور المضغوطة فئه

الكلمات المفتاحية: تمييز الوجه، تحليل العنصر الاساسي، تحلل القيمة المفردة،المويجات المتقطعة هار ، مسافة منهاتن 